\title{
CONCAVE RESOURCE ALLOCATION PROBLEMS FOR INTERFERENCE COUPLED WIRELESS SYSTEMS
}

\author{
Holger Boche, Siddharth Naik \\ Technical University of Berlin \\ Heinrich Hertz Institute (HHI) \\ Einsteinufer 25, 10587 Berlin \\ holger.boche@mk.tu-berlin.de \\ naik@hhi.fraunhofer.de
}

\author{
Tansu Alpcan \\ Technical University of Berlin \\ Deutsche Telekom \\ Laboratories \\ Ernst-Reuter Platz 7, 10587 Berlin \\ alpcan@sec.t-labs.tu-berlin.de
}

\begin{abstract}
The paper characterizes the class of all concave resource allocation problems in interference coupled wireless systems. An axiomatic framework for interference functions proposed by Yates in 1995 is used to model interference coupling in our paper. The paper shows that there exists no transformation, which ensures concavity for all linear interference functions for all functions of SINR. The paper then characterizes the largest class of utility functions under a certain requirement, such that the corresponding class of utility functions functions, which are a function of SINR in the $s$-domain are concave. The paper shows that such a class of utility functions is a restricted class due to a requirement, which ensures concavity. Furthermore, the paper shows that the largest class of interference functions, which ensures concavity for resource allocation problems are the log-convex interference functions. These results differ from the convex case, where we are interested in minimizing utility functions of inverse SINR.
\end{abstract}

Index Terms - Resource allocation, interference coupled systems, utility maximization

\section{INTRODUCTION}

In wireless systems, we frequently encounter resource allocation problems, which are functions of the signal-to-(noise) plus interference ratio (SINR). We are often in a situation, where we would like to maximize certain functions of SINR - to improve the system performance, e.g. rate, capacity. It would be helpful for such functions to be concave or be transformed into concave functions in a certain domain. It is strongly believed, that the dividing line between "easy" and "difficult" problems in optimization is convexity. Investigation of concavity properties of functions of SINR are significantly different from investigating the convexity properties of function, which are functions of inverse SINR. In the convex case, we would like to minimize the functions of inverse SINR. We shall elucidate the differences between the concave and convex cases, throughout the paper.

We characterize the class of resource allocation problems, which can be written as concave optimization problems, thereby establishing inherent boundaries on concavity properties of functions in interference coupled wireless systems. We adopt an axiomatic approach to model interference in our system. An axiomatic approach was proposed by Yates in [1] with extensions in [2,3]. The Yates framework of standard interference functions (discussed in detail in Section 2.2) is general enough to incorporate cross-layer effects and it serves as a theoretical basis for a plethora of algorithms, e.g. beamforming [4]
[5] states that there exists no SINR based utility functions, which are convex or concave in the power domain. Furthermore, the sum of such weighted functions can never be convex or concave. In this paper, we attempt to obtain concavity of certain resource allocation problems, under an appropriate transformation. The main contributions of this paper are as follows:

- Under very natural assumptions, we present an impossibility result (Theorem 1), which states that there exists no transformation $\psi$, such that the function $\psi\left(s_{k}\right) / \mathcal{I}_{k}(\psi(s))$ for all users $k$ is jointly concave with respect to $s$, where $\mathcal{I}_{k}(\psi(s))$ is an interference function (more details in Section 5).

- Theorem 2 establishes the largest class of utility functions $(\mathcal{E C}$ onc $)$, which are functions of SINR in the $s$-domain and are concave. Due to a certain requirement of the theorem (explained in detail in Lemma 1) such a class of utility functions is a restricted class. Furthermore, it is shown that the family of exponential functions is the unique family of functions, such that a relevant and frequently encountered function in interference coupled wireless systems is jointly concave for all linear interference functions and for all utility functions in the class $\mathcal{E}$ Conc.

- Theorem 3 proves that the largest class of interference functions, which preserves concavity of resource allocation strategies of interference coupled wireless systems is the family of log-convex interference functions.

\section{INTERFERENCE COUPLED WIRELESS SYSTEMS}

In this paper we shall investigate the case of interference coupled wireless systems. We begin, by providing certain notational conventions used in the paper in Section 2.1 below.

\subsection{Preliminaries and Notation}

Matrices and vectors are denoted by bold capital letters and bold lowercase letters, respectively. Let $\boldsymbol{y}$ be a vector, then $y_{l}=[\boldsymbol{y}]_{l}$ is the $l^{\text {th }}$ component. The notation $y \geq 0$ implies that $y_{l} \geq 0$ for all components $l$. $\boldsymbol{x}>\boldsymbol{y}$ implies $x_{l}>y_{l}$ for all components $l$. Similar definitions hold for the reverse directions. $\boldsymbol{x} \neq \boldsymbol{y}$ implies that the vector differs in at least one component. Let $\mathcal{F}$ imply a set, with the exception that $\mathcal{I}$ is used to represent a function. The set of nonnegative reals is denoted as $\mathbb{R}_{+}$. The set of positive reals is denoted as $\mathbb{R}_{++}$. Let $e^{\boldsymbol{y}}$ and $\log (\boldsymbol{y})$ denote component-wise exponential and logarithm, respectively. Let $g, f$ and $\psi$ represent functions throughout the paper. 


\subsection{Interference Functions}

In a wireless system, the users' utilities can strongly depend on the underlying physical layer. An important measure for the link performance is the SINR. Consider $K$ users with transmit powers $\boldsymbol{p}=$ $\left[p_{1}, \ldots, p_{K}\right]^{T}$ and $\mathcal{K}:=\{1, \ldots, K\}$. The noise power at each receiver is $\sigma^{2}$. Hence the SINR at each receiver depends on the extended power vector $\boldsymbol{p}=\left[\boldsymbol{p}, \sigma^{2}\right]^{T}=\left[p_{1}, \ldots, p_{K}, \sigma^{2}\right]^{T}$. The resulting SINR of user $k$ is $\operatorname{SINR}_{k}(\underline{\boldsymbol{p}})=\frac{p_{k}}{\mathcal{I}_{k}(\underline{\boldsymbol{p}})}=\gamma_{k}(\underline{\boldsymbol{p}})$, where $\mathcal{I}_{k}$ is the interference (plus noise) as a function of $\boldsymbol{p}$. In order to model interference coupling, we shall follow the axiomatic approach proposed in $[1,3]$. The general interference functions possess the properties of conditional positivity, scale invariance and monotonicity with respect to the power component and strict monotonicity with respect to the noise component. For further details, kindly refer to the Appendix 5 .

\subsection{Impact of Interference Coupling}

Users in a wireless systems coupled by interference are intrinsically competitive. Each of them is principally interested in maximizing their own utility and have little or no regard for the utilities of the other users and for the entire system utility. Such neglect of course does not come for free. Such a characterization is accompanied by a pre-condition that there must be at least one user $k \in \mathcal{K}$ who sees interference from another user $j \in \mathcal{K}$ and $j \neq k$, i.e. it must not be possible to completely orthogonalize all the users in the system. If the users are completely orthogonalized, then they are coupled only by the constraints on the resource allocation strategy and there is no "competition" in the sense as we describe in this section.

Example 1. Consider the function $u_{k}(\underline{\boldsymbol{p}})=\log \left(\underline{p}_{k} / \mathcal{I}_{k}(\underline{\boldsymbol{p}})\right)$. The corresponding problem of maximizing the function $f(\underline{\boldsymbol{p}}, \boldsymbol{\omega})=$ $\sum_{k \in \mathcal{K}} \omega_{k} u_{k}(\underline{\boldsymbol{p}})$ is the often encountered weighted proportional fairness problem. Function $f(\boldsymbol{p}, \boldsymbol{\omega})$ is not jointly concave with respect to $\boldsymbol{p}$, for all non-orthogonal systems of users, for all weight vectors $\overline{\boldsymbol{\omega}}>\mathbf{0}$. Function $f(\underline{\boldsymbol{p}}, \boldsymbol{\omega})$ is not jointly concave even for fixed linear interference functions, e.g. $\mathcal{I}_{k}(\underline{\boldsymbol{p}})=\sum_{l \in \mathcal{K}} v_{k l} \underline{p}_{l}+\theta_{k}^{2}$, where $v_{k l}$ is the link-gain between transmitter $l$ and receiver $k$.

[5] states that if $u_{k}$ is the rate of user $k$, then the following sum of weighted rate maximization problem is not concave optimization problem with respect to $\boldsymbol{p}$ for all non-orthogonal system of users. We now investigate the possibility of suitably transforming the problem, so as to obtain a concave objective function for resource allocation problems in Section 3.

\section{ANALYSIS OF CONCAVITY PROPERTIES OF RESOURCE ALLOCATION PROBLEMS}

In this section, we shall analyze concavity properties of functions of SINR. Before we delve into our analysis, we shall briefly review the concepts of feasible SINR regions and feasible quality-of-service (QoS) regions. The feasible SINR region $\mathbf{F}$ is the set of all feasible SINR vectors $\bar{\gamma}$, that can be supported for all users by means of power control, with interference being treated as noise. We define $\mathcal{P}$ as the set of vectors, which satisfy certain power constraints, e.g.

- for the case of total power constraints: $\mathcal{P}:=\{\boldsymbol{p} \mid \boldsymbol{p}=$ $\left.\left[p_{1}, \ldots, p_{K}\right], \sum_{k \in \mathcal{K}} p_{k} \leq P_{\text {total }}\right\}$, where $P_{\text {total }}$ is the total power constraint,

- for the case of individual power constraints: $\mathcal{P}:=\{\boldsymbol{p} \mid \boldsymbol{p} \leq$ $\hat{\boldsymbol{p}}\}$, where $\hat{\boldsymbol{p}}=\left[\hat{p}_{1}, \ldots, \hat{p}_{K}\right]$ are the individual power constraints, and
- for the case of individual and total power constraints: $\mathcal{P}:=$ $\left\{\boldsymbol{p} \mid \boldsymbol{p}=\left[p_{1}, \ldots, p_{K}\right], \sum_{k \in \mathcal{K}} p_{k} \leq P_{\text {total }}, \boldsymbol{p} \leq \hat{\boldsymbol{p}}\right\}$.

The feasible SINR region $\mathbf{F}$ can be written as follows:

$$
\left.\mathbf{F}=\left\{\bar{\gamma} \mid \exists \underline{\boldsymbol{p}} \geq \mathbf{0}, \boldsymbol{p} \in \mathcal{P}, \gamma_{k}(\underline{\boldsymbol{p}})\right) \geq \bar{\gamma}_{k}, \bar{\gamma}_{k} \in \mathbb{R}_{+}, \forall k \in \mathcal{K}\right\}
$$

and the corresponding feasible QoS region is

$$
\mathbf{U}=\left\{\overline{\boldsymbol{u}} \mid \exists \boldsymbol{\gamma} \in \mathbf{F}, u_{k}\left(\gamma_{k}(\underline{\boldsymbol{p}})\right) \geq \bar{u}_{k}, \bar{u}_{k} \in \mathbb{R}_{+}, \forall k \in \mathcal{K}\right\} .
$$

We know from [6] and the references there in, that the feasible SINR region $(\mathbf{F})$ is in general not convex ${ }^{1}$. Furthermore, we also know from [5], that we can never have joint concavity of the SINR in the power domain. Hence, we would like to investigate the possibility of finding a suitable transformation $\psi$ (or $\psi^{-1}$ ), which

1. transforms the problem from the power domain to the $s-$ domain, i.e. $\psi^{-1}: \mathbb{R}_{+} \mapsto S$, where $S=\mathbb{R}, s \in S$ and the inverse SINR and functions of inverse SINR are jointly convex wrt. $s=\left[s_{1}, \ldots, s_{K}\right]$,

2. transforms the feasible SINR region into a convex feasible QoS set $\mathbf{U}$, where $\psi^{-1}\left(\gamma_{k}\right)=u_{k}$, for all $k \in \mathcal{K}$ and $\boldsymbol{u} \in \mathbf{U}$.

While finding our transformation $\psi^{-1}$, we shall assume that: transformation $\psi(s)=p$ is strictly monotonic increasing and twice continuously differentiable, throughout the paper.

The feasible SINR region is convex on the logarithmic scale ${ }^{2}$ We now return to problem of finding a suitable transformation $\psi$. To formalize the conditions 1) and 2) we introduce the following requirement.

Requirement 1. For all linear interference functions, the supportable QoS region $\mathbf{U}$ resulting from the transformation $\gamma_{k}=\psi\left(u_{k}\right)$, $\boldsymbol{u}=\left[u_{1}, \ldots, u_{K}\right] \boldsymbol{u} \in \mathbf{U}$ without power constraints is convex.

Having formalized our requirement we begin by analyzing the concavity properties of functions of SINR for linear interference functions in Section 3.1.

\subsection{Analysis of Concavity Properties of Resource Allocation Problems - Linear Interference Functions}

Linear interference functions are the simplest type of interference functions and they are frequently encountered in communication systems. Hence, expecting the supportable QoS region to be convex for all linear interference functions, is a very natural requirement for communication systems. We shall present an impossibility result (Theorem 1), which has implications on the concavity properties of resource allocation strategies, aiming to maximizing functions of SINR in interference coupled wireless systems. We briefly deviate and look at the convex case, where we have a completely different picture. In the convex case, we have to analyze the function $\mathcal{I}_{k}(\psi(s)) / \psi\left(s_{k}\right)$ - since the convex case is connected with "loss minimization". It can be observed, that $\psi(s)=e^{\mu s}$, with $\mu>0$ always leads to jointly convex behaviour with respect to $s$ (for all linear interference functions).

\footnotetext{
${ }^{1}$ The feasible SINR region can also be defined as $\{\boldsymbol{\gamma}>\mathbf{0} \mid \rho(\boldsymbol{\gamma}) \leq$ $1\}$, where $\rho(\boldsymbol{\gamma}):=\rho\left(\operatorname{diag}\{\boldsymbol{\gamma}\} \boldsymbol{V}_{\text {res }}\right)$ is the spectral radius of the weighted coupling matrix, where $\boldsymbol{V}=\left[\boldsymbol{V}_{\text {res }}, \mathbf{1}^{T}\right]$ and $\boldsymbol{V}_{\text {res }}$ is an irreducible matrix containing the interference coupling coefficients, without the dependency on noise.

${ }^{2}$ This is due to the fact that the spectral radius $\rho(\gamma)$ is log-convex after a change of variable $\gamma=e^{s}$, where $s \in \mathbb{R}^{K+1}$ is the logarithmic SINR.
} 
Theorem 1. There exists no strictly monotonic increasing and twice continuously differentiable transformation $\psi$, such that for all linear interference functions, the function $\psi\left(s_{k}\right) / \mathcal{I}_{k}(\psi(s))$, for all $k \in \mathcal{K}$ is jointly concave with respect to $s$.

Proof. For the sake of obtaining a contradiction, assume that the statement of the Theorem 1 is not true, i.e. there exists such a function. Choose $\mathcal{I}_{k}(\underline{\boldsymbol{p}})=\sum_{j \in \mathcal{K} \backslash k} v_{j} \underline{p}_{j}$ and fix $s_{j}$, for all $j \in \mathcal{K} \backslash k$. Then the function $\psi\left(s_{k}\right) / \sum_{j \in \mathcal{K} \backslash k} v_{j} \psi\left(s_{j}\right)$ is concave with respect to $s_{k}$, i.e. the transformation $\psi$ is itself concave.

Now, fix $s_{1}$ (w.l.o.g) and consider the following expression $\psi\left(s_{1}\right) / \psi\left(s_{2}\right)$, with $s_{2} \in \mathbf{S} \subset \mathbb{R}$. This implies, that $1 / \psi\left(s_{2}\right)$ is a concave function. Now, choose $s_{2}^{(1)}, s_{2}^{(2)} \in \mathbf{S}, s_{2}^{(1)} \neq s_{2}^{(2)}$ arbitrarily. We have, that $\psi\left(s_{2}^{(1)}\right) \neq \psi\left(s_{2}^{(1)}\right)$. Take, $s_{2}(\lambda):=$ $(1-\lambda) s_{2}^{(1)}+\lambda s_{2}^{(2)}$. Then, we have that

$$
\frac{1}{\psi\left(s_{2}(\lambda)\right)} \geq(1-\lambda) \frac{1}{\psi\left(s_{2}^{(1)}\right)}+\lambda \frac{1}{\psi\left(s_{2}^{(2)}\right)} .
$$

On the other hand, we have that (since $\psi$ is concave) $\psi\left(s_{2}(\lambda)\right) \geq$ $(1-\lambda) \psi\left(s_{2}^{(1)}\right)+\lambda \psi\left(s_{2}^{(2)}\right)$. This give us the following expression:

$$
\begin{aligned}
\frac{1}{\psi\left(s_{2}(\lambda)\right)} & \leq \frac{1}{(1-\lambda) \psi\left(s_{2}^{(1)}\right)+\lambda \psi\left(s_{2}^{(2)}\right)} \\
& <(1-\lambda) \frac{1}{\psi\left(s_{2}^{(1)}\right)}+\lambda \frac{1}{\psi\left(s_{2}^{(2)}\right)} .
\end{aligned}
$$

The strict inequality above follows from the strict convexity of $1 / x$ and we have our required contradiction with (3).

Remark 1. We have proved the statement of the Theorem 1, for the case, when we can scale the noise. Similarly, we can easily prove the Theorem for the noise free case. We shall now analyze the case, when we have noise and we do not scale the noise. Consider the function

$$
\frac{\psi\left(s_{k}\right)}{\sum_{j \in \mathcal{K} \backslash k} v_{k j} \psi\left(s_{j}\right)+v_{k(K+1)} \sigma^{2}} .
$$

As $v_{k(K+1)} \rightarrow 0$, we have that the function (4) tends to the noise free case. However, we have already stated, that with the above proof technique we can prove the Theorem for the noise free case. Furthermore, we know that the limit of a sequence of concave functions is concave. Since, we have that the limit function here is not concave, we can conclude that the individual sequences are not concave either.

We have observed, that the concavity of $g$, e.g. $g(x)=x$ is not sufficient to ensure the joint concavity of $g\left(\psi\left(s_{k}\right) / \mathcal{I}_{k}(\psi(s))\right)$, $k \in \mathcal{K}$ with respect to $s$ for a certain transformation. Hence, we need to restrict utility functions $g$, such that we can ensure joint concavity of the desired function. The necessary condition, which ensures joint concavity will be presented in Lemma 1 below.

Lemma 1. Let a strictly monotonic increasing and twice continuously differentiable function $\psi$ satisfy requirement 1 . Let $g$ be a monotonic increasing function. Let $g\left(\psi\left(s_{k}\right) / \mathcal{I}_{k}(\psi(s))\right)$, for all $k \in \mathcal{K}$ be jointly concave with respect to $s$ for a certain $\psi$ and for all linear interference functions $\mathcal{I}_{1}, \ldots, \mathcal{I}_{K}$. Then, $g\left(e^{x}\right)$ is convave.

Proof. Since requirement 1 is satisfied, from Theorem 1 in [6] we have that $\psi$ is log-convex. Choose $x_{1}, x_{2} \in \mathbb{R}$ arbitrarily. Then, we have for $x(\lambda)=(1-\lambda) x_{1}+\lambda x_{2}$, that $\psi(x(\lambda)) \leq$ $\left(\psi\left(x_{1}\right)\right)^{1-\lambda}\left(\psi\left(x_{2}\right)\right)^{\lambda}$. Since $g$ is monotonic increasing, we have that $g(\psi(x(\lambda))) \leq g\left(\left(\psi\left(x_{1}\right)\right)^{1-\lambda}\left(\psi\left(x_{2}\right)\right)^{\lambda}\right)$. Since $g(\psi(x))$ is concave, we have that $(1-\lambda) g\left(\psi\left(x_{1}\right)\right)+\lambda g\left(\psi\left(x_{2}\right)\right) \leq$ $g\left(\psi\left(x_{1}\right)^{1-\lambda} \psi\left(x_{2}\right)^{\lambda}\right)$. Now, let $y_{1}, y_{2} \in \mathbb{R}$ be arbitrarily chosen. We choose $x_{1}, x_{2}$ such that $\psi\left(x_{k}\right)=e^{y_{k}}$, for $k \in\{1,2\}$. This is possible due to our assumption on $\psi$. Let $y(\lambda)=(1-\lambda) y_{1}+\lambda y_{2}$, then we have that $(1-\lambda) g\left(e^{y_{1}}\right)+\lambda g\left(e^{y_{2}}\right) \leq g\left(e^{y(\lambda)}\right)$, i.e. $g\left(e^{y}\right)$ is concave.

We have observed that to ensure the joint concavity of the function $g\left(\psi\left(s_{k}\right) / \mathcal{I}_{k}(\psi(s))\right)$ with respect to $s$, for all $k \in \mathcal{K}$, concavity of $g\left(e^{x}\right)$ is a necessary condition. Since, $0 \geq\left(g\left(e^{y}\right)\right)^{\prime \prime}=$ $\left(g^{\prime}\left(e^{y}\right) e^{y}\right)^{\prime}=g^{\prime \prime}\left(e^{y}\right) e^{2 y}+g^{\prime}\left(e^{y}\right) e^{y}$. We have, that if $g$ is strictly monotonic increasing, then $g(x)$ is also concave, i.e. the concavity of the function $g\left(e^{y}\right)$ is a stronger requirement. To utilize this requirement, we introduce the the family of functions $\mathcal{E C}$ onc below.

Definition 1. $\mathcal{E}$ Conc is the family of all strictly monotonic increasing, continuous functions $g$, such that $g\left(e^{x}\right)$ is concave.

We have seen in Lemma 1 , the existence of a function $\psi$ such that the function $g\left(\psi\left(s_{k}\right) / \mathcal{I}_{k}(\psi(s))\right)$, for all $k \in \mathcal{K}$ is jointly concave with respect to $s$, for all functions $g \in \mathcal{E C}$ onc. We shall now show in Theorem 2, that the function $\psi=c_{1} \exp (\mu s)$, is up to two constants $c_{1}, \mu$ the unique transformation, which ensures the joint concavity of $g\left(\psi\left(s_{k}\right) / \mathcal{I}_{k}(\psi(s))\right)$, for all linear interference functions and for all utility functions $g \in \mathcal{E C}$ onc.

We briefly compare this situation with the convex case, i.e. minimizing the function $g\left(\mathcal{I}_{k}(s) / \psi\left(s_{k}\right)\right)$, where $\psi$ is the exponential function. For the case of linear interference functions and for all strictly monotonic increasing, continuous and convex functions $g$, we have that $g\left(\mathcal{I}_{k}(s)\right) / \psi\left(s_{k}\right)$ is jointly convex with respect to $s$. In this case we do not require any further restrictions.

Theorem 2. For all linear interference functions $\mathcal{I}_{1}, \ldots, \mathcal{I}_{K}$ and for all $g \in \mathcal{E}$ Conc, the function $g\left(\psi\left(s_{k}\right) / \mathcal{I}_{k}(\psi(s))\right)$ is jointly concave with respect to $s$, if and only if $\psi(s)=c_{1} \exp (\mu s)$, with $c_{1}, \mu>0$.

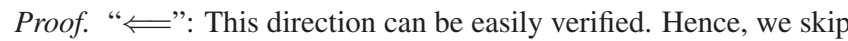
the proof.

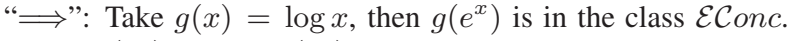
Now, take $g\left(s_{k}\right)=\log \psi\left(s_{k}\right)$, then $g$ is in accordance with our assumption, hence is concave. However, the function $\psi$ is also $\log$ convex and let $s(\lambda)=(1-\lambda) s_{1}+\lambda s_{2}$ and choose $s_{1}=0, s_{2}=1$, then we have that

$$
\begin{aligned}
\log \psi(s(\lambda)) & =\log \psi(\lambda)=(1-\lambda) \log \psi(0)+\lambda \log \psi(1) \\
& =\log \psi(0)+\lambda\left(\log (\underbrace{\frac{\psi(1)}{\psi(0)}}_{>1})\right)
\end{aligned}
$$

Let $\psi(1) / \psi(0)=\mu$, then we have that $\psi(\lambda)=\psi(0) e^{\lambda \mu}$, where $\mu>1$.

We now extend our insight obtained from Theorem 1 and the Lemma 1, beyond linear interference functions.

\subsection{Analysis of Concavity Properties of Resource Allocation Problems - Beyond Linear Interference Functions}

In this section, we shall analyze the concavity properties of resource allocation problems for interference functions, beyond the class of linear interference functions. An example of non-linear interference functions is worst case interference: $\mathcal{I}_{k}(\boldsymbol{p})=\max _{c_{k} \in \mathcal{C}_{k}} \boldsymbol{p}^{T} \boldsymbol{v}_{k}\left(c_{k}\right)$, for $k \in \mathcal{K}$. The parameter $c_{k}$ can represent some uncertainty, chosen from a compact uncertainty set $\mathcal{C}_{k}$. Such worst case interference functions are often used in the case of robust power control. 
We shall be particularly interested in investigating the following problem: We would like to preserve the concavity of the function $g\left(\psi\left(s_{k}\right) / \mathcal{I}_{k}(\psi(s))\right)$, for all $k \in \mathcal{K}$ for the "largest" class of interference functions. Therefore, we shall check for the concavity of the function $\sum_{k \in \mathcal{K}} \omega_{k} g\left(\psi\left(s_{k}\right) / \mathcal{I}_{k}(\psi(s))\right)$ for all weight vectors $\boldsymbol{\omega}>\mathbf{0}$, for all utility functions $g \in \mathcal{E} \mathcal{C}$ onc for the largest possible class of interference functions. Then, we have the following result.

Theorem 3. The function $\sum_{k \in \mathcal{K}} \omega_{k} g\left(\psi\left(s_{k}\right) / \mathcal{I}_{k}(\psi(s))\right)$ is jointly concave with respect to $s$, for all weight vectors $\boldsymbol{\omega}>\mathbf{0}$, for all $g \in \mathcal{E C}$ onc, if and only if $\mathcal{I}_{1}, \ldots, \mathcal{I}_{K}$ are log-convex interference functions.

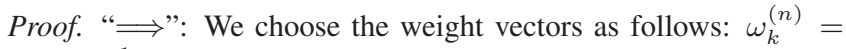
$\left\{\begin{array}{cc}1-\frac{1}{n} & k=j \\ \frac{1}{(K-1) n} & k \neq j\end{array}\right.$ Taking the limit as $n \rightarrow \infty$, we have that

$$
g\left(\frac{\psi\left(s_{j}\right)}{\mathcal{I}_{j}(\psi(s))}\right)=\lim _{n \rightarrow \infty} \sum_{k \in \mathcal{K}} \omega_{k}^{(n)} g\left(\frac{\psi\left(s_{k}\right)}{\mathcal{I}_{k}(\psi(s))}\right) .
$$

We achieve that $g\left(e^{s_{k}} / \mathcal{I}_{k}\left(e^{s}\right)\right):=G(s)$ is jointly concave with respect to $s$, for all $g \in \mathcal{E} \mathcal{C}$ onc. Since, the limit function of a sequence of concave function is concave. Choose $g(x)=\log (x)$, then for $g \in \mathcal{E C}$ onc we have that $\log \mathcal{I}_{k}\left(e^{s}\right)=s+\log G_{k}(s)$, i.e. $\mathcal{I}_{k}\left(e^{s}\right)$ is log-convex.

" $\Longleftarrow$ ": If interference functions $\mathcal{I}_{1}, \ldots, \mathcal{I}_{K}$ are log-convex, then for $s^{(1)}, s^{(2)}$ arbitrarily chosen and $s(\lambda):=(1-\lambda) s^{(1)}+\lambda s^{(2)}$, we have that

$$
\begin{aligned}
& \mathcal{I}_{k}\left(e^{s(\lambda)}\right) \leq\left(\mathcal{I}_{k}\left(e^{s^{(1)}}\right)\right)^{1-\lambda}\left(\mathcal{I}_{k}\left(e^{s^{(2)}}\right)\right)^{\lambda} \\
& \text { i.e. } \frac{e^{s_{k}(\lambda)}}{\mathcal{I}_{k}\left(e^{s(\lambda)}\right)} \geq\left(\frac{e^{s_{k}^{(1)}}}{\mathcal{I}_{k}\left(e^{s^{(1)}}\right)}\right)^{1-\lambda}\left(\frac{e^{s_{k}^{(2)}}}{\mathcal{I}_{k}\left(e^{s^{(2)}}\right)}\right)^{\lambda} .
\end{aligned}
$$

Then, for $g \in \mathcal{E C}$ onc fixed, we have that

$$
\begin{aligned}
g\left(\frac{e^{s_{k}(\lambda)}}{\mathcal{I}_{k}\left(e^{s(\lambda)}\right)}\right) & \geq g\left(\left(\frac{e^{s_{k}^{(1)}}}{\mathcal{I}_{k}\left(e^{s^{(1)}}\right)}\right)^{1-\lambda}\left(\frac{e^{s_{k}^{(2)}}}{\mathcal{I}_{k}\left(e^{s^{(2)}}\right)}\right)^{\lambda}\right) \\
& \geq(1-\lambda) g\left(\frac{e^{s_{k}^{(1)}}}{\mathcal{I}_{k}\left(e^{s^{(1)}}\right)}\right)+\lambda g\left(\frac{e^{s_{k}^{(2)}}}{\mathcal{I}_{k}\left(e^{s^{(2)}}\right)}\right)(.6)
\end{aligned}
$$

Inequality in (6) is obtained, since $g\left(e^{y}\right)$ is concave. Hence, we have that $g\left(e^{s_{k}} / \mathcal{I}_{k}\left(e^{s}\right)\right)$ is jointly concave with respect to $s$, for all $k \in$ $\mathcal{K}$. Hence, we have that $\sum_{k \in \mathcal{K}} \omega_{k} g\left(\psi\left(s_{k}\right) / \mathcal{I}_{k}(\psi(s))\right)$ is jointly concave for all weight vectors $\boldsymbol{\omega}>\mathbf{0}$ and for all $g \in \mathcal{E}$ Conc.

We contrast the result obtained from Theorem 3 to the convex case. In the convex case, i.e. minimization of the function $\sum_{k \in \mathcal{K}} \omega_{k} g\left(\mathcal{I}_{k}(\psi(s)) / \psi\left(s_{k}\right)\right)$, where $g$ is a strictly monotonic increasing, continuous and convex function - it can be observed that with log-convex interference functions (have been discussed in [7]) and with $\psi$ as the exponential transformation, $\sum_{k \in \mathcal{K}} \omega_{k} g\left(\mathcal{I}_{k}(\psi(s)) / \psi\left(s_{k}\right)\right)$ is jointly convex with respect to $s$.

\section{DISCUSSION}

Many resource allocations problems encountered in wireless systems have objective functions, which are functions of SINR, e.g. rate maximization. For all non-orthogonal systems, i.e. all non-trivial interference functions, these objective functions are never jointly concave in the power domain. Our paper has explored the possibility of obtaining a suitable transformation, such that the resulting resource allocation problem is jointly concave. Our paper proves, that there exists no transformation, which ensures joint concavity, for all functions of SINR, for all linear interference functions. Hence, we require additional restrictions to ensure concavity of the desired functions. The paper has quantified this requirement and identified the largest class of utility functions $\mathcal{E}$ Conc, which are a function of SINR in the $s$-domain and always jointly concave for linear interference functions. Furthermore, it is shown that the family of exponential functions is the unique family of functions, such that a relevant and frequently encountered function in interference coupled wireless systems is jointly concave for all linear interference functions and for all utility functions in the class $\mathcal{E}$ Conc. We have shown that the largest class of interference functions, which ensures concavity for resource allocation strategies are the log-convex interference functions.

\section{APPENDIX: INTERFERENCE FUNCTIONS}

Definition 2. Interference functions: We say that $\mathcal{I}: \mathbb{R}_{+}^{K+1} \mapsto \mathbb{R}_{+}$ is an interference function if the following axioms are fulfilled:

A1 conditional positivity $\mathcal{I}(\underline{\boldsymbol{p}})>0$ if $\underline{\boldsymbol{p}}>\mathbf{0}$

$A 2 \quad$ scale invariance $\mathcal{I}(\alpha \underline{\boldsymbol{p}})=\alpha \mathcal{I}(\underline{\boldsymbol{p}}), \forall \alpha \in \mathbb{R}_{+}$

A3 monotonicity $\mathcal{I}(\boldsymbol{p}) \geq \mathcal{I}(\hat{\boldsymbol{p}})$ if $\underline{\boldsymbol{p}} \geq \hat{\boldsymbol{p}}$

A4 strict monotonicity $\mathcal{I}(\underline{\boldsymbol{p}})>\mathcal{I}(\underline{\hat{\boldsymbol{p}}})$ if $\underline{\boldsymbol{p}} \geq \underline{\hat{\boldsymbol{p}}}$,

$$
\underline{p}_{K+1}>\underline{\hat{p}}_{K+1} \text {. }
$$

Note that we require that $\mathcal{I}(\underline{\boldsymbol{p}})$ is strictly monotone with respect to the last component $\underline{p}_{K+1}$, e.g. $\mathcal{I}(\underline{\boldsymbol{p}})=\boldsymbol{v}^{T} \boldsymbol{p}+\sigma^{2}$, where $v \in \mathbb{R}_{+}^{K}$ is a vector of interference coupling coefficients. The axiomatic framework A1-A4 is connected with the framework of standard interference functions [1]. The details about the relationship between the model A1-A4 and Yates' standard interference functions were discussed in [3]. We note that the results of this paper are also applicable to standard interference functions.

\section{REFERENCES}

[1] R. D. Yates, "A Framework for Uplink Power Control in Cellular Radio Systems," IEEE Journal on Selected Areas in Communication, vol. 13, no. 7, 1995.

[2] C. Huang and R. Yates, "Rate of Convergence for Minimum Power Assignment Algorithms in Cellular Radio Systems," Baltzer/ACM Wireless Networks, vol. 4, pp. 223-231, 1998.

[3] Martin Schubert and Holger Boche, QoS-Based Resource Allocation and Transceiver Optimization, vol. 2, now Publishers Inc., Boston-Delft, 2006.

[4] D. Hammarwall, M. Bengtsson, and B. Ottersten, "On Downlink Beamforming with Indefinite Shaping Constraints," IEEE Transactions on Signal Processing, vol. 54, pp. 3566-3580, September 2006.

[5] Holger Boche and Siddharth Naik, "Impact of Interference Coupling - Loss of Convexity," in IEEE Globecom, Honolulu, Hawaii, USA, December 2009.

[6] Slavomir Stanczak and Holger Boche, "On the Convexity of Feasible QoS Regions," IEEE Transactions on Information Theory, vol. 53, no. 2, pp. 779-783, February 2007.

[7] Holger Boche and Martin Schubert, "Complete Characterization of the Pareto Boundary of Interference Coupled Wireless Systems with Power Constraints - the Log-Convex Case," in ICASSP, Taipeh, Taiwan, April 2009. 